\title{
Sensitivities of outdoor sound propagation predictions to environmental input parameters
}

\author{
François-Edern Aballéa, ${ }^{\text {a) }}$ Jérôme Defrance, ${ }^{\text {b) }}$ Marine Baulac, ${ }^{\text {b) }}$ Matthieu Fournier ${ }^{c}$ \\ and Eric Premat ${ }^{\mathrm{d})}$
}

(Received 2006 January 26; revised 2006 July 17; accepted 2006 July 18)

\begin{abstract}
A number of reference numerical models can be used to perform outdoor sound propagation prediction in a complex environment. Most of time, either they consume little computational time by not taking all phenomena into account at the same time, or they are more complete but their calculation times are prohibitive. This paper presents some principles which can be used in reference numerical models to reduce calculation times in predicting long-range outdoor sound propagation under complex environments. Limits, assumptions and approximations used to predict outdoor sound propagation are discussed here in terms of their impact on accuracy for typical road traffic configurations as a function of frequency, geometry of the site and atmospheric conditions.

(C) 2007 Institute of Noise Control Engineering.
\end{abstract}

Primary subject classification: 24; Secondary subject classification: 75

\section{INTRODUCTION}

Noise from road and railway infrastructures are being more severely regulated by European laws: acceptable thresholds in emission and reception are decreasing. It becomes essential to obtain more accurate tools to predict long-range acoustic waves propagation in the atmosphere. This implies the need be able to take many phenomena into account at the same time, e.g. meteorology, uneven ground and impedance discontinuities. Some reference models have been developed. On one hand, the more complete models consider most of phenomena but their long calculations times became a concern. On the other hand, models with low, more acceptable computational times ignore some phenomena.

a) Now at Ecole Polytechnique Federale de Lausanne (EDFL), STI ITOP LEMA, Station 11, CH-1015 Lausanna SWITZERLAND; email: francois.abellea@epfl.ch; Centre Scientifique et Technique du Bâtiment (CSTB), Acoustics and Lighting Department, 24, rue Joseph Fourier, 38400 Saint Martin d'Hères, FRANCE; email: f.aballea@cstb.fr

b) Centre Scientifique et Technique du Bâtiment (CSTB), Acoustics and Lighting Department, 24, rue Joseph Fourier, 38400 Saint Martin d'Hères, FRANCE.

c) Centres d'Etudes Techniques de l'Equipement (CETE) de Lyon, Boulevard de l'industrie, BP 141, 71404 Autun Cedex, FRANCE.

d) Centres d'Etudes Techniques de l'Equipement (CETE) de Lyon, 46, rue Saint-Théobald, BP 128, 38081 L'Isle d'Abeau cedex, FRANCE.
The railway configuration presented in Fig. 1 is an example of a complex situation which is difficult to handle from every angle. To obtain precise predictions of the sound pressure levels produced by the train, the acoustician had to employ a sophisticated model able to consider at least:

-- discontinuities in ground impedance

-- multiple reflections created by the two parallel barriers

-- $\quad$ the range dependant meteorology

-- $\quad$ the aspect of the linear railway source

However such models are seldom used because their calculation time is truly prohibitive.

The aim of the paper is to present assumptions to reduce calculation time by reference models used in sound propagation prediction in a complex environment, and to overcome some of their limitations. By

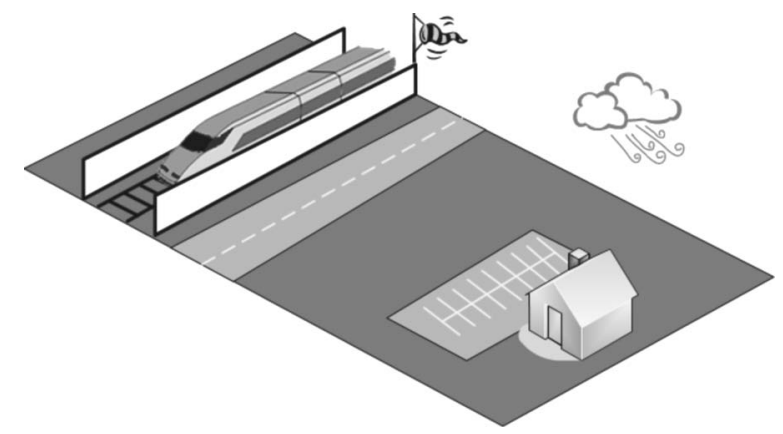

Fig. 1-Example of a typical complex configuration with impedance jump, reflections, 3 dimensional effects and meteorology. 
'reference model' we mean numerical methods that give 'exact' results for a specific configuration. Putting these assumptions into practice will produce results from reference models which could be useful in the development of new engineering methods or to undertake parametric studies.

Several effects are addressed in this work:

a) the application of an average ground instead of a mixed ground for ground impedance description,

b) the importance of the order of reflection,

c) the error due to the use of a range independent wind speed profile instead of a range-dependent wind speed profile,

d) the use of a two-dimensional (2D) model instead a three-dimensional (3D) model,

e) a method allowing the use of a linear sound speed profile in place of a logarithmic sound speed profile.

In this paper, the influence of each of these on road traffic noise are explored using two computational codes in this paper: ATMOS ${ }^{1,2}$ (Advanced Theoretical Models for Outdoor Sound propagation), a Parabolic Equation (PE) computational code conjointly developed by CSTB and CEA (Commissariat de l'Energie Atomique), and $\mathrm{MICADO}^{3}$ (Integral Method for Acoustical Calculations of Diffractions by Obstacles), a Boundary Element Method (BEM) computational code developed in CSTB.

Part of this work was performed during the European Harmonoise Project. ${ }^{4}$

\section{NUMERICAL MODEL}

\subsection{Description of the Parabolic Equation (PE) Method}

$\operatorname{ATMOS}^{2,3}$ is a PE based numerical code. The PE is an approximation to the Helmholtz wave equation and is one of the most powerful numerical methods effective for long-range, forward-wave propagation thought relatively general media. ${ }^{5,6}$ Introduced at the beginning of the 1940s in order to solve electromagnetism problems, this theory has been applied to ocean acoustics and then to atmospheric sound propagation. ${ }^{7}$ One of the PE developments, called GFPE (Green Function Parabolic Equation), was adapted for the atmospheric propagation by Gilbert and $\mathrm{Di}^{8,9}$ at the beginning of the 1990s.

A known starting field is propagated step by step up to the receiver. The ground impedance and the sound speed profile may vary for each step with a low cost in calculation time. The great advantage of this method is that steps of computations may be considerably larger than the wavelength instead of a fraction of a wavelength for classical PE; the consequence is a significant decrease of computation time.

Using the $e^{-i \omega t}$ convention the elliptic form of the Helmholtz equation for the harmonic sound pressure $p$ in $(r, z)$ coordinates can be rewritten:

$$
\left(\frac{\partial^{2}}{\partial r^{2}}+\frac{1}{r} \frac{\partial}{\partial r}+\frac{\partial^{2}}{\partial z^{2}}+k^{2}(r, z)\right) p(r, z)=0
$$

with $k(r, z)=\frac{\omega}{c(r, z)}$ the effective wave number, $c(r, z)$ the effective sound speed and $\omega$ the angular frequency.

Denoting $p(r, z)=\frac{1}{\sqrt{r}} \phi(r, z) e^{j k_{r} r}$ and neglecting the azimuthal derivative of the field in the wave equation, the equation of propagation Eqn. (1) can be the written, for $k r \gg 1$ :

$$
\frac{\partial^{2} \phi(r, z)}{\partial r^{2}}+\frac{\partial^{2} \phi(r, z)}{\partial z^{2}}+k(r, z)^{2} \phi(r, z)=0
$$

The relative sound speed gradient is assumed to vary slowly with distance, so that it is assumed on a given range step that the wave number $k$ depends only on height $z$. Neglecting backscattering, a solution of Eqn. (2) for a range independent sound speed profile in a step is:

$$
\phi(r+\Delta r, z)=e^{j \Delta r \sqrt{Q}} \phi(r, z)
$$

where the operator $Q$ is written:

$$
Q=\frac{\partial^{2}}{\partial z^{2}}+k^{2}(r, z)
$$

In a given step, the square of the total wave number is split into a constant reference wave number $k_{r}^{2}$ at zero height and a small contribution $\delta k^{2}$ that varies with height:

$$
k^{2}(z)=k_{r}^{2}+\delta k^{2}(z)
$$

After many developments described by Gilbert ${ }^{8}$ and Di and Salomons, ${ }^{10}$ the field at $\phi(r+\Delta r, z)$ is given by:

$$
\begin{aligned}
\phi(r+\Delta r, z)= & {\left[\frac{1}{2 \pi} \int_{-\infty}^{+\infty}\left(\Phi\left(r, k^{\prime}\right)+R\left(k^{\prime}\right) \Phi\left(r-k^{\prime}\right)\right)\right.} \\
& \times e^{j \Delta r\left(\sqrt{k_{r}^{2}-k^{\prime 2}}-k_{r}\right)} e^{j k^{\prime} z} d k^{\prime}+2 j \beta \times \Phi(r, \beta) \\
& \left.\times e^{j \Delta r\left(\sqrt{k_{r}^{2}-\beta^{2}}-k_{r}\right)} e^{-j \beta z}\right] \times e^{j \frac{\Delta r \delta k^{2}(z)}{2 k_{r}}}
\end{aligned}
$$

with $\Phi(r, z)=\int_{0}^{\infty} \phi(r, z) e^{-j k z^{\prime}} d z$ the Fourier transform of $\phi$.

$\beta=-k_{r} / Z_{g}$ represents the surface wave pole in the reflecting coefficient $R\left(k^{\prime}\right)$ :

$$
R\left(k^{\prime}\right)=\frac{k^{\prime} Z_{g}-k_{r}}{k^{\prime} Z_{g}+k_{r}}
$$

where $Z_{g}$ is the normalized ground impedance. 


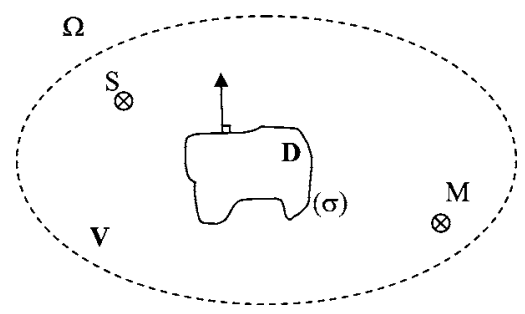

Fig. 2-General scheme of Green representation. $\Omega$ is the space surrounding the volume $D$ of the boundary $\sigma . V$ is a volume which includes the volume $D$, the source $S$ and any point $M$.

Equation (6) is the product of an exponential factor $\Delta r \delta k^{2}(z)$

$e^{j \frac{j k_{r}}{2 k_{r}}}$, which represents the contribution of the non-constant sound speed profile, and of 3 terms $\Phi\left(r, k^{\prime}\right), R\left(k^{\prime}\right) \Phi\left(r,-k^{\prime}\right)$ and $\Phi(r, \beta)$ representing the direct wave, the wave reflected by the ground and the surface wave respectively. Details about the numerical implementation of Eqn. (6) are discussed by Salomons ${ }^{10}$ and Barrière ${ }^{11}$ and Gabillet.

\subsection{Description of the Boundary Element Method (BEM)}

MICADO is a numerical code based on the BEM. This approach relies on the Integral Equation theory which was developed in the 1960s and has been since extensively used. ${ }^{12}$ Its main advantage is that it allows any kind of shape and absorption of the surfaces to be taken into account for in a homogeneous atmosphere.

In this approach, the two variables to determine are speed and pressure. The acoustic field $P$ is solution of the Helmholtz equation:

$$
\left(\nabla^{2}+k^{2}\right) P(r, z)=f(r, z) \quad \forall(r, z) \in \Omega
$$

where $f(r, z)$ is the source distribution, $k$ the wave number and $\Omega$ the domain surrounding a volume $D$ whose boundary is $\sigma$ (Fig. 2).

Using the Green's function and the Sommerfeld condition, the following formula is obtained after mathematical simplifications: ${ }^{12}$

$$
\begin{aligned}
\Pi(r, z) P(r, z)= & P_{0}(r, z)+\int_{\sigma}\left[P(r, z) \frac{\partial G}{\partial n_{s}}\left(r_{0}, z_{0}, r, z\right)\right. \\
& \left.-G\left(r_{0}, z_{0}, r, z\right) \frac{\partial P}{\partial n_{s}}(r, z)\right] d S \\
& \forall(r, z) \in \Omega
\end{aligned}
$$

with coefficient $\Pi$ depending on the receiver position.

In MICADO a variational approach ${ }^{3}$ is used to solve Eqn. (9). The geometry of the problem is 2D: the source is an infinite coherent linear source and all the configurations considered remain unchanged and infinite along a direction perpendicular to the vertical section plane. The ground as well as any obstacle surface are reflective or can be characterized by their own acoustical admittance $\alpha$. The theoretical formalism relies on an integral representation of the pressure field at any point as a function of the pressure on the boundaries, the admittances of the boundaries, as well as the Green's solution $G$ (elementary solution for a point source $M$ and for a receiver $N$ above an impedance plane) which can be written as the sum of three different terms:

$$
G(M, N)=-\frac{i}{4} H_{0}(k r)-\frac{i}{4} H_{0}\left(k r^{\prime}\right)+P_{\alpha}(M, N)
$$

where $r$ is the distance between source and receiver, $r^{\prime}$ the distance between the image-source and the receiver and $H_{0}$ the Hankel function of first kind and zero order. The second term in Eqn. (10) represents the contribution of the reflection of the cylindrical wave on a perfectly rigid ground. The last term $P_{\alpha}$ is a corrective factor which takes into account the ground admittance.

Duhamel ${ }^{13}$ and Jean et al. ${ }^{14}$ have presented a way to compute the pressure field of a line source by post-processing $2 \mathrm{D}$ results. Using the representation of a 3D point source in terms of integral Hankel functions, they show it is possible to approximate pressure field in $3 \mathrm{D}$ from $2 \mathrm{D}$ pressure results. This approach is called 2D1/2-BEM.

\section{GROUND EFFECTS}

\subsection{Introduction}

The case of more than one type of ground surface is often encountered in outdoor sound propagation. Most of time two types of surfaces have to be considered: an absorbing surface made of soil covered with vegetation (grass, plants) and a reflective surface made of asphalt, concrete or stone. The change of surface type does not necessarily correspond to the change of topography. Moreover, along a path, there may be many successive different surface types at the same level. We study here the possibility of using an equivalent absorbing ground surface in place of two different surfaces.

The calculations are achieved in a homogeneous atmosphere using MICADO. The two types of ground are modelled here by two impedances: an infinite one for the rigid case, and one calculated with the Delany and Bazley's mode ${ }^{15}$ with an air flow resistivity of $200 \mathrm{kPa} . \mathrm{s} . \mathrm{m}^{-2}$. The first simulations concern a ground made of $50 \%$ of reflective and $50 \%$ of absorbing parts. A few more calculations are also presented for $33 \%$ reflective and $67 \%$ absorbing. 


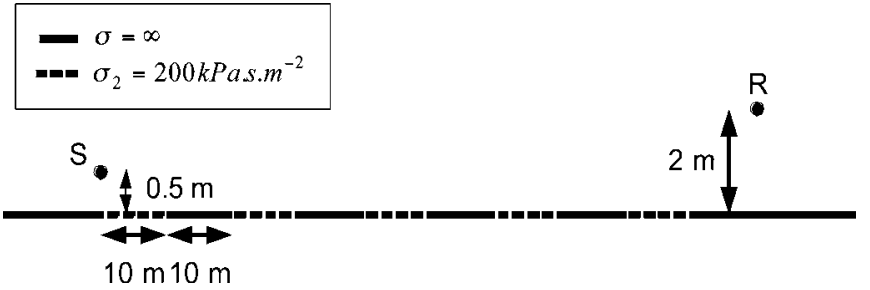

Fig. 3-Example of configuration (case 8). The ground between the source $S$ and the receiver $R$ is divided in regular reflective and absorbing successive strips of $10 \mathrm{~m}$.

\subsection{Configurations and Results}

The monopole source is $0.5 \mathrm{~m}$ high. The receiver is $2 \mathrm{~m}$ high and $100 \mathrm{~m}$ away from the source (horizontal distance). The "100 m" of propagation are divided in regular reflective and absorbing successive strips (Fig. 3 ). Ground "before" source and "after" receiver is rigid.

The calculations are performed from 50 to $5000 \mathrm{~Hz}$ with 10 frequencies per third octave band. Different cases are studied. For quite small strips:

- Case 1: $0.5 \mathrm{~m}$ wide strips (first strip "after" source is reflective),

- Case 2: $0.5 \mathrm{~m}$ wide strips (first strip "after" source is absorbing),

- Case 3: $1 \mathrm{~m}$ wide strips (first strip "after" source is reflective),

- Case 4: $1 \mathrm{~m}$ wide strips (first strip "after" source is absorbing),

For wider strips:

(a)

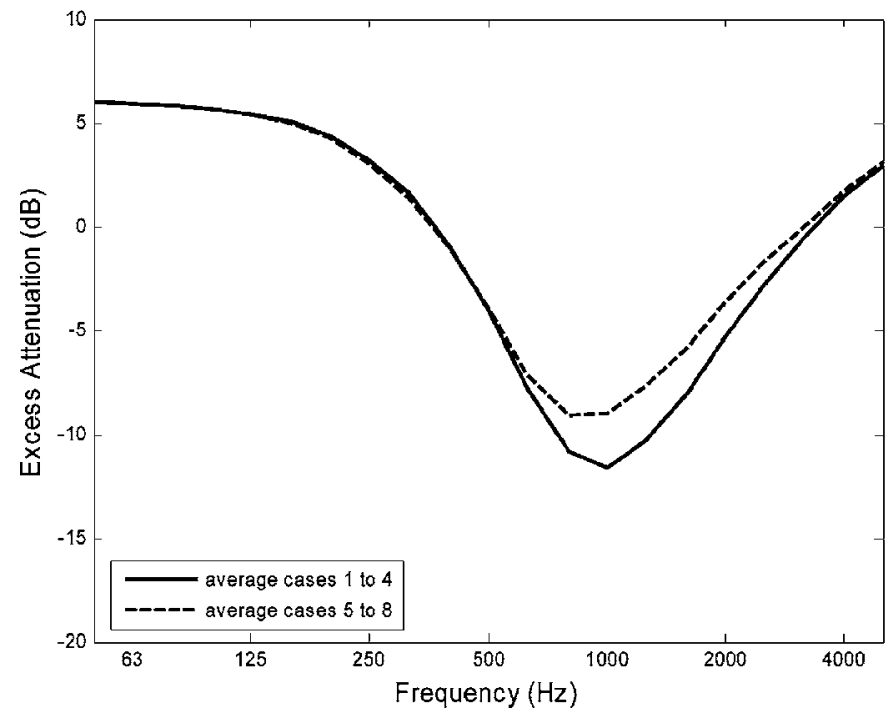

- Case 5: $5 \mathrm{~m}$ wide strips (first strip "after" source is reflective),

- Case 6: $5 \mathrm{~m}$ wide strips (first strip "after" source is absorbing),

- Case 7: $10 \mathrm{~m}$ wide strips (first strip "after" source is reflective),

- Case 8: $10 \mathrm{~m}$ wide strips (first strip "after" source is absorbing),

Another couple of configurations have been investigated with strips of alternating widths $1 \mathrm{~m}$ and $2 \mathrm{~m}$.

- $\quad$ Case 9: 1 and $2 \mathrm{~m}$ successive wide strips (first $1 \mathrm{~m}$ strip is absorbing, i.e. $33 \%$ of the surface is absorptive),

- $\quad$ Case 10: 1 and $2 \mathrm{~m}$ successive wide strips (first $1 \mathrm{~m}$ strip is reflective, ie. $67 \%$ of the surface is absorptive)

The results are given in terms of excess attenuation as a function of frequency in Fig. 4 and Fig. 5. For each set of cases, the closest equivalent resistivity $\sigma_{e q}$ is calculated by dichotomy for each octave band mid frequency as follows: for each band, two resistivities $\sigma_{1}$ and $\sigma_{2}$ are first chosen to obtain two excess attenuations $E A_{1}$ and $E A_{2}$ bordering the reference excess attenuation $E A_{\text {ref. }}$ A new excess attenuation $E A_{3}$ is calculated using a resistivity $\sigma_{3}$, which is the mid-value of the interval $\left[\sigma_{1} ; \sigma_{2}\right]$. Afterward, the subinterval containing $E A_{\text {ref }}$ is subdivided using the same principle as described above until the wanted accuracy is obtained. Equivalent resistivities for each set of cases are given in Table 1 and Table 2 (rounded by $10 \mathrm{kPa} . \mathrm{s} . \mathrm{m}^{-2}$ ).

(b)

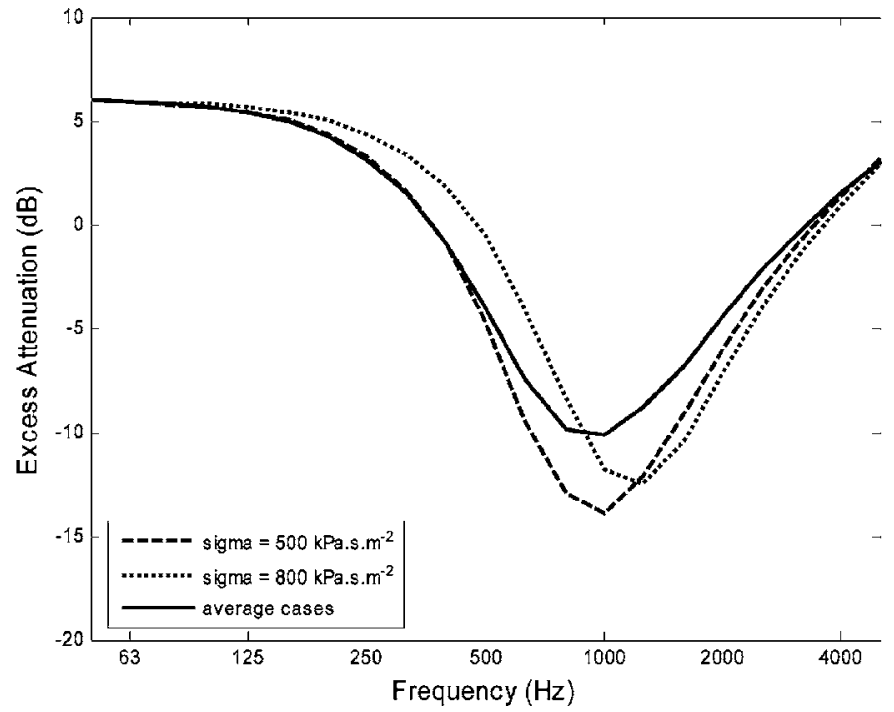

Fig. 4-Average Excess Attenuation as a function of frequency a) for cases 1 to 4 and 5 to 8 and b) for cases 1 to 8 compared to two different homogeneous ground configurations results. 
(a)

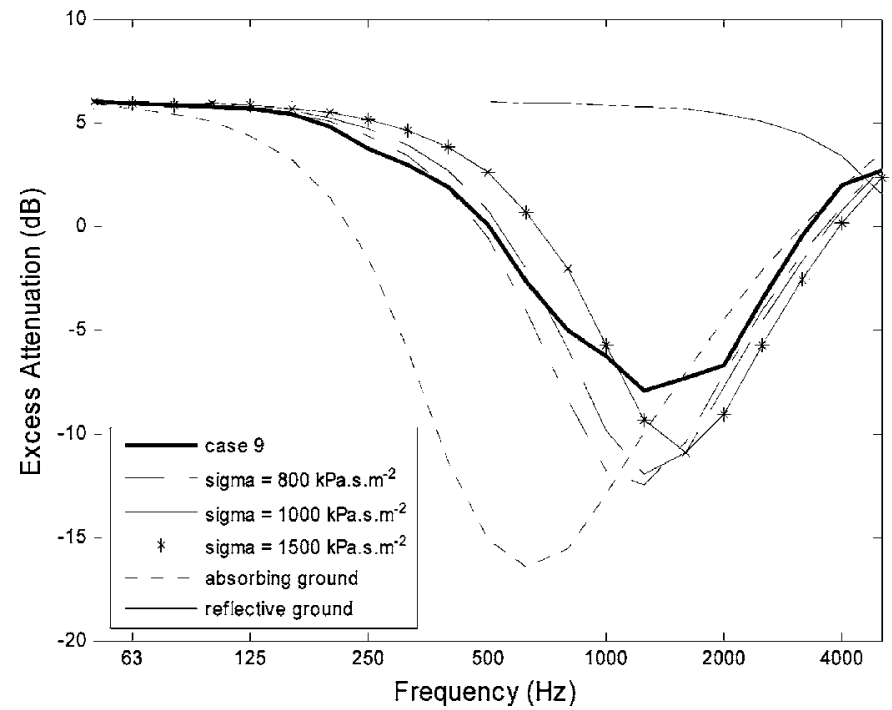

(b)

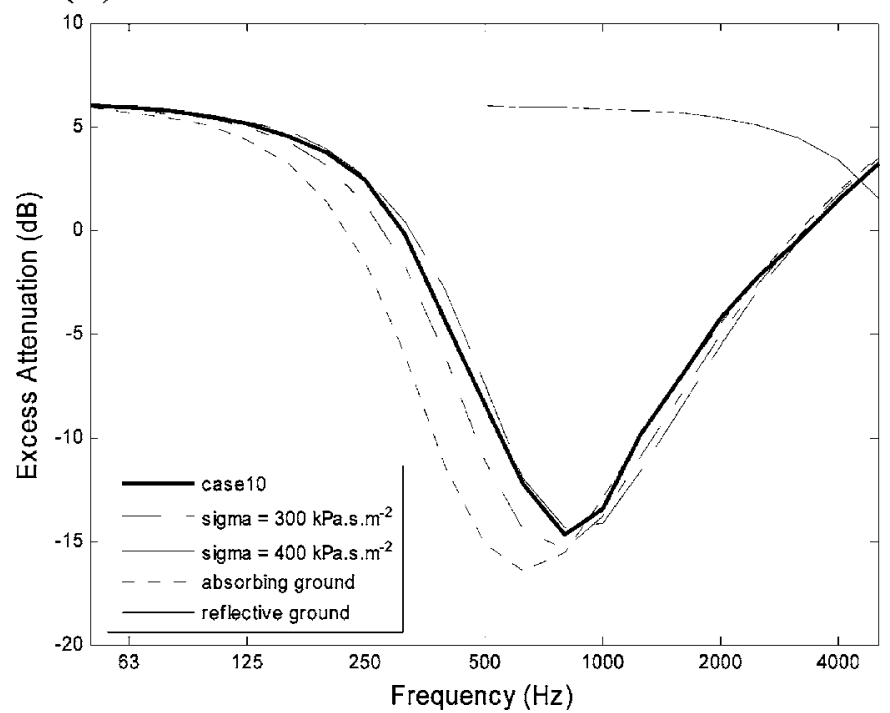

Fig. 5-Excess attenuation as a function of frequency a) for case $9(33 \%$ abs) and b) for case 10 (67\% abs) compared to different homogeneous ground configurations results.

\subsection{Discussion and Conclusions}

Table 2 gives the trends of equivalent values of resistivity as a function of percentage of absorbing parts. Those average values have been obtained from the configurations described above with several heights of receiver and source. Results given in Fig. 4 and Fig. 5 show that an equivalent absorbing ground can be used as a good approximation to a complex ground with impedance jumps. For a normalized sound spectrum ${ }^{16}$
(Table 3), difference in the A-weighted global excess attenuations calculated for the real case and with the average ground method does not exceed $0.3 \mathrm{~dB}$.

This approach can be useful to reduce calculation time in some reference models. For example, calculation time of BEM can be decreased by a factor of 5 for a propagation distance of $100 \mathrm{~m}$ to a factor 100 for a propagation distance of $1000 \mathrm{~m}$ by the use of a homogeneous equivalent ground surface as a substitute

Table 1 -Closest equivalent resistivity $\sigma_{e q}$ calculated by dichotomy (rounded by $10 \mathrm{kp} . \mathrm{a} . \mathrm{s} . \mathrm{m}^{-2}$ ) for each octave band mid frequency.

\begin{tabular}{lccccc}
\hline \hline & \multicolumn{5}{c}{$\sigma_{\text {eq }}\left(\mathrm{kPa}\right.$ s. $\mathrm{m}^{-2}$ ) } \\
\cline { 2 - 6 } Freq. (Hz) & Cases 1 to 4 & Cases 5 to 8 & Cases 1 to 8 & Case 9 & Case 10 \\
\hline 125 & 500 & 470 & 480 & $>5000$ & 340 \\
250 & 480 & 460 & 470 & 590 & 380 \\
500 & 530 & 540 & 540 & 890 & 370 \\
1000 & 820 & 1090 & 970 & 1420 & 250 \\
2000 & 360 & 70 & 180 & 670 & 140 \\
\hline \hline
\end{tabular}

Table 2-Equivalent values of resistivity $\sigma_{\mathrm{eq}}$ as a function of percentage of absorbing parts.

\begin{tabular}{lcc}
\hline \hline $\begin{array}{l}\text { Percentage of } \\
\text { absorbing parts }\end{array}$ & 125,250 and $500 \mathrm{~Hz}$ & 1000 and $2000 \mathrm{~Hz}$ \\
\hline $100 \%$ & $\sigma_{\mathrm{eq}}=200 \mathrm{kPa} \cdot \mathrm{s} \cdot \mathrm{m}^{-2}$ & $\sigma_{\mathrm{eq}}=200 \mathrm{kPa} \cdot \mathrm{s} \cdot \mathrm{m}^{-2}$ \\
$67 \%$ & $\sigma_{\mathrm{eq}}=400 \mathrm{kPa} \cdot \mathrm{s} \cdot \mathrm{m}^{-2}$ & $\sigma_{\mathrm{eq}}=300 \mathrm{kPa} \cdot \mathrm{s} \cdot \mathrm{m}^{-2}$ \\
$50 \%$ & $\sigma_{\mathrm{eq}}=500 \mathrm{kPa} \cdot \mathrm{s} \cdot \mathrm{m}^{-2}$ & $\sigma_{\mathrm{eq}}=800 \mathrm{kPa} \cdot \mathrm{s} \cdot \mathrm{m}^{-2}$ \\
$33 \%$ & $\sigma_{\mathrm{eq}}=800 \mathrm{kPa} \cdot \mathrm{s} \cdot \mathrm{m}^{-2}$ & $\sigma_{\mathrm{eq}}=1500 \mathrm{kPa} \cdot \mathrm{s} \cdot \mathrm{m}^{-2}$ \\
$0 \%$ & $\sigma_{\mathrm{eq}}=\infty$ & $\sigma_{\mathrm{eq}}=\infty$ \\
\hline \hline
\end{tabular}


Table 3-Normalized sound spectrum for traffic.

\begin{tabular}{cccccccccc}
\hline \hline Frequency & $100 \mathrm{~Hz}$ & $125 \mathrm{~Hz}$ & $160 \mathrm{~Hz}$ & $200 \mathrm{~Hz}$ & $250 \mathrm{~Hz}$ & $315 \mathrm{~Hz}$ & $400 \mathrm{~Hz}$ & $500 \mathrm{~Hz}$ & $630 \mathrm{~Hz}$ \\
$\mathrm{~L}(\mathrm{~dB})$ & -20 & -20 & -18 & -16 & -15 & -14 & -13 & -12 & -11 \\
Frequency & $800 \mathrm{~Hz}$ & $1000 \mathrm{~Hz}$ & $1250 \mathrm{~Hz}$ & $1600 \mathrm{~Hz}$ & $2000 \mathrm{~Hz}$ & $2500 \mathrm{~Hz}$ & $3150 \mathrm{~Hz}$ & $4000 \mathrm{~Hz}$ & $5000 \mathrm{~Hz}$ \\
$\mathrm{~L}(\mathrm{~dB})$ & -9 & -8 & -9 & -10 & -11 & -13 & -15 & -16 & -18 \\
\hline \hline
\end{tabular}

to a mixed ground surface. This method can also be used by some reference methods such as FFP (Fast Field Program), which does not allow for an impedance jump along the propagation path.

\section{MULTIPLE REFLECTION EFFECTS}

\subsection{Introduction}

In this approach, backscattering created by reflections from a vertical obstacle is considered with a PE model by using a complementary Kirchhoff approximation called GFPE-Kirchhoff. ${ }^{17}$ This method allows for the solution of a multiple reflection problem by choosing the order of reflection. We investigate here the effect of the reflection order. Calculations are achieved in an homogeneous atmosphere using ATMOS and compared with MICADO. MICADO gives an exact solution which corresponds to an infinite order of reflections. Since it is a BEM approach it doesn't permit the possibility of choosing the reflection order.

\subsection{Configuration and Results}

A source located between two vertical barriers is studied. The geometry and the calculation principle for an order of reflection of 2 are described in Fig. 6. The calculation principle comes from the image-source theory where image-sources are created relative to barrier vertical plane. For all reflections, the sound pressure at any calculation point above the obstacle is set to zero and then propagated to the receiver. For an order of reflection of 2 , the total pressure at the receiver is the sum of 3 fields: (a) diffracted, (b) simply reflected and diffracted, and (c) double reflected and diffracted. Results at the order 1, 6 and 20 are compared to MICADO calculation in Fig. 7.

\subsection{Discussion and Conclusions}

Results show the importance of the number of reflections to take into account to reach an acceptable convergence of the prediction. However, this number is not easy to determine since it depends both on configuration and frequency: it has to be increased when the two vertical barriers get closer or when the frequency increases. This is the case in our results. If only 6 reflections are sufficient to reach convergence for frequencies lower than $1000 \mathrm{~Hz}, 20$ are recommended for higher frequencies. However, for an emission spectrum $^{16}$ of a normalized traffic noise (Table 3), the difference in A-weighted global excess attenuation between 6 and 20 reflections is less than $0.5 \mathrm{~dB}$ while the time of calculation is divided by 4 . With this small

(A)

(B)

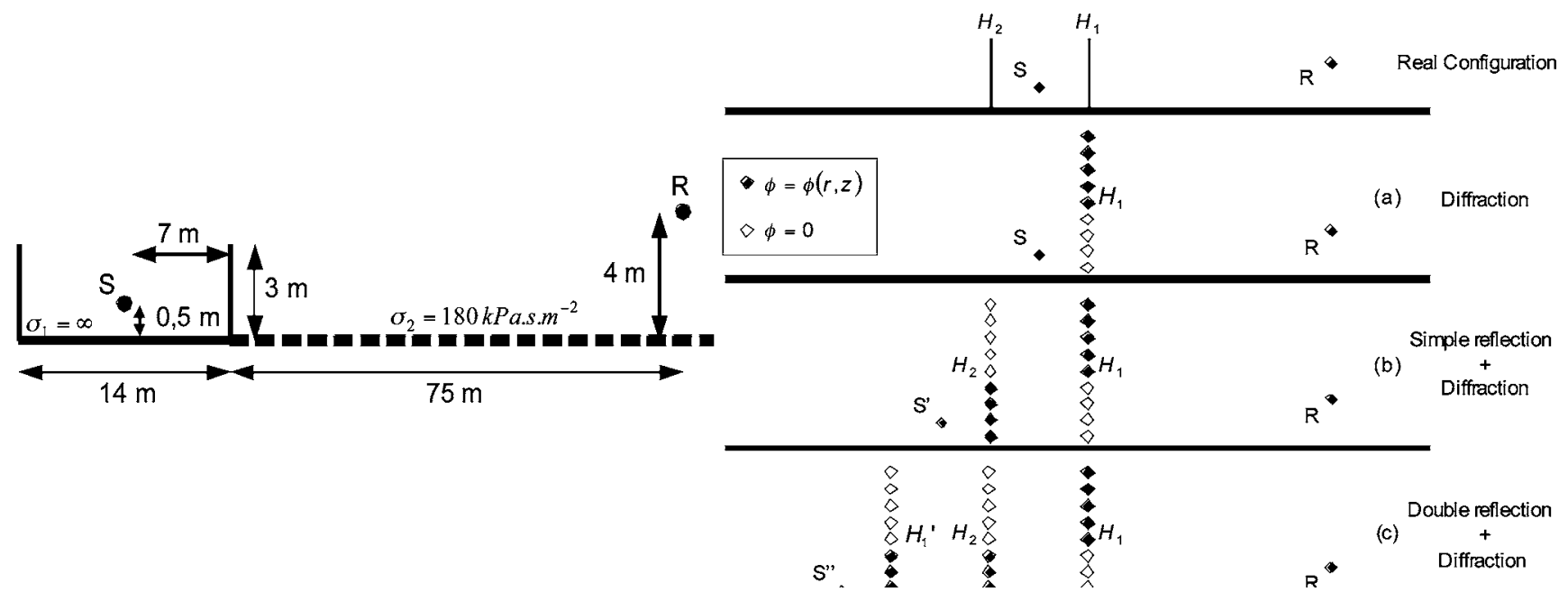

Fig. 6-A) Road traffic geometry and B) principle of calculation at the order of 2 . The total pressure at the receiver is the sum of 3 fields: (a) diffracted (from $S$ ), (b) simply reflected and diffracted (from $\left.S^{\prime}\right)$ and double reflected and diffracted (from $\left.S^{\prime \prime}\right)$ 


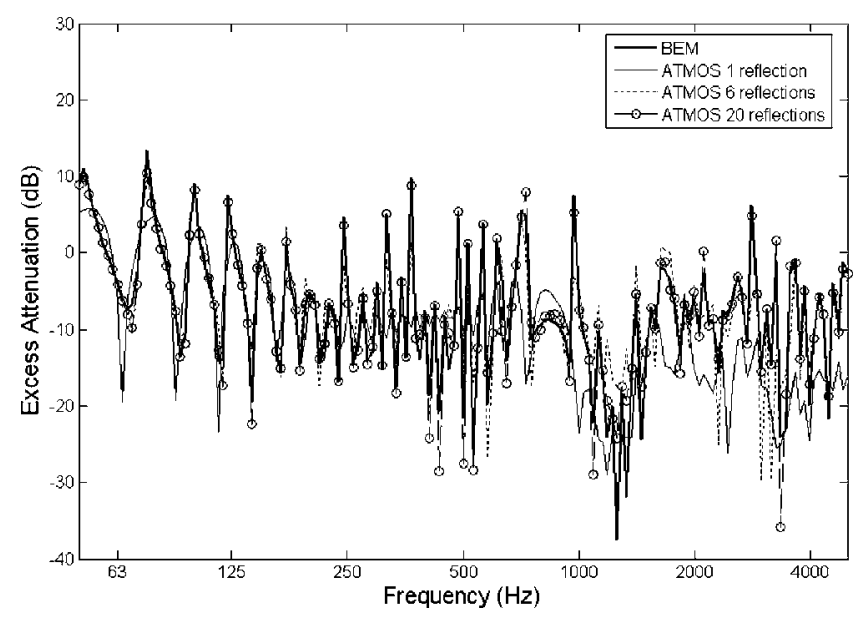

Fig. 7-Excess attenuation as a function of frequency for different reflection order.

error, it is then possible to reduce the number of reflection to decrease calculation times without significantly sacrificing accuracy.

\section{WIND EFFECT OVER BARRIERS}

\subsection{Introduction}

The sound speed profile in real traffic noise situations usually varies due to topography and atmospheric instabilities. However, most of reference models use a range independent sound speed profile. We study here the effect of this assumption for a barrier. Calculations are achieved in inhomogeneous atmosphere using ATMOS. Wind speed distribution over the barrier is computed with FLUENT, a Computational Fluid Dynamics (CFD) code.

\subsection{Configuration and Results}

We use an initial logarithmic sound speed profile:

$$
c(z)=c_{0}+v(z)
$$

where $c_{0}$ is the reference sound speed and $v(z)$ the horizontal wind speed component given by:

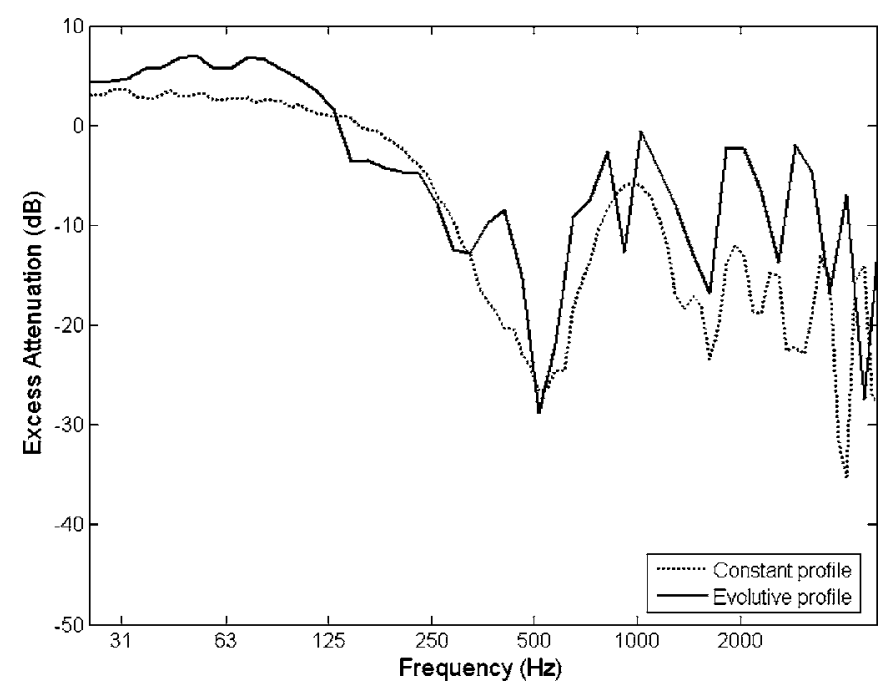

Fig. 9-Excess attenuation as a function of frequency for range independent and range dependent wind speed profiles.

$$
v(z)=b \ln \left(1+\frac{z}{z_{0}}\right)
$$

with $b=1.12 \mathrm{~ms}^{-1}$ the refractive index and $z_{0}$ $=2.91 .10^{-5} \mathrm{~m}$ the roughness length. This wind, which corresponds to a wind speed of $14 \mathrm{~m} . \mathrm{s}^{-1}$ at $10 \mathrm{~m}$ above the ground is used as FLUENT input. Road traffic configuration and FLUENT results are presented in Fig. 8. Excess attenuation for range independent and range dependent wind speed profile is given in Fig. 9.

\subsection{Discussion and Conclusion}

The two main effects of the presence of a barrier on wind speed profiles are: the increase of the wind velocity above the barrier (sort of Venturi effect) and the recirculation behind the obstacle (Fig. 8). These modifications in the wind profile leads to high sound speed gradients which usually decrease the barrier efficiency (Fig. 9). Thus, the difference in the A-weighted global excess attenuation for a normalized road traffic emission spectrum ${ }^{16}$ (Table 3 ) is about

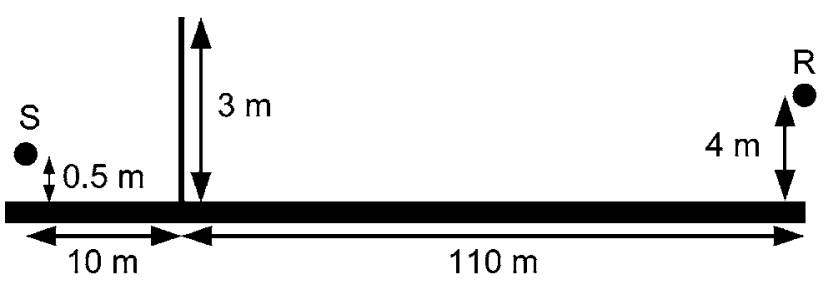

(a)

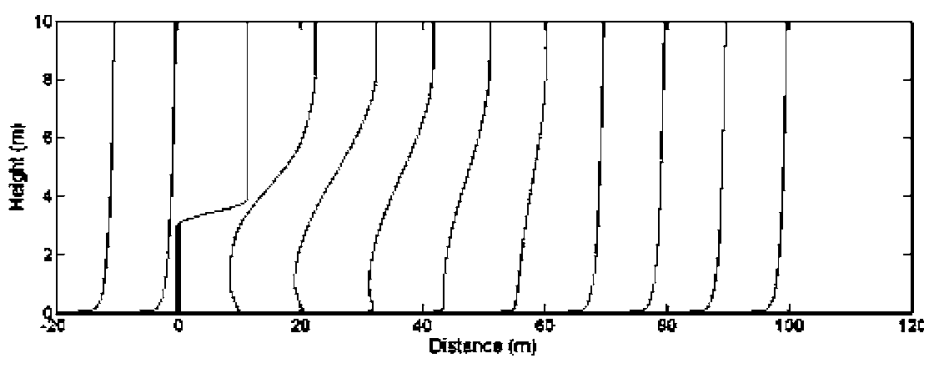

(b)

Fig. 8-a) Geometry of the road traffic configuration and b) evolution of the sound speed profile computed with FLUENT. 


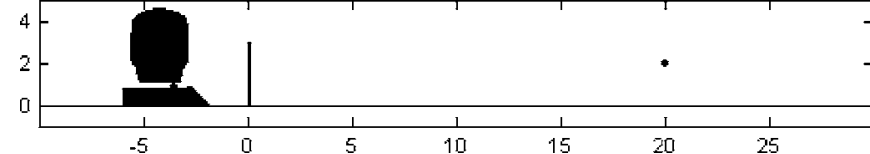

Fig. 10-Geometry of the configuration. $S(-3.5,0.85)$ and $R(20.0,2.0)$.

$3 \mathrm{~dB}$ lower for the range-independent than for the range-dependent sound speed profile. The significant induced error points out the importance of taking wind speed profile evolution into account in the evaluation of barrier efficiency.

\section{OBLIQUE PROPAGATION OVER A BARRIER}

\subsection{Introduction}

In this part, we study the possibility of using a series of $2 \mathrm{D}$ calculations to take into account real 3D geometry in the case of a railway noise. Even if

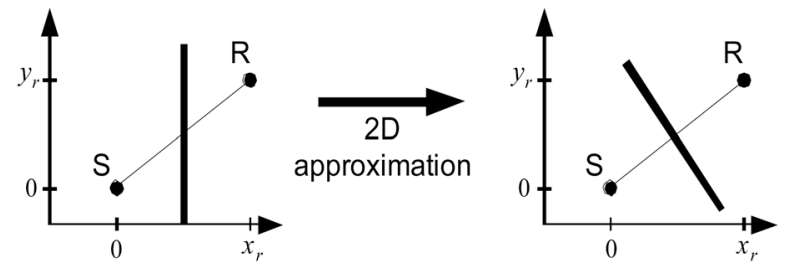

Fig. 11-Illustration of a two-dimensional approximation for oblique propagation over a barrier. The two figures are top views.

(a)

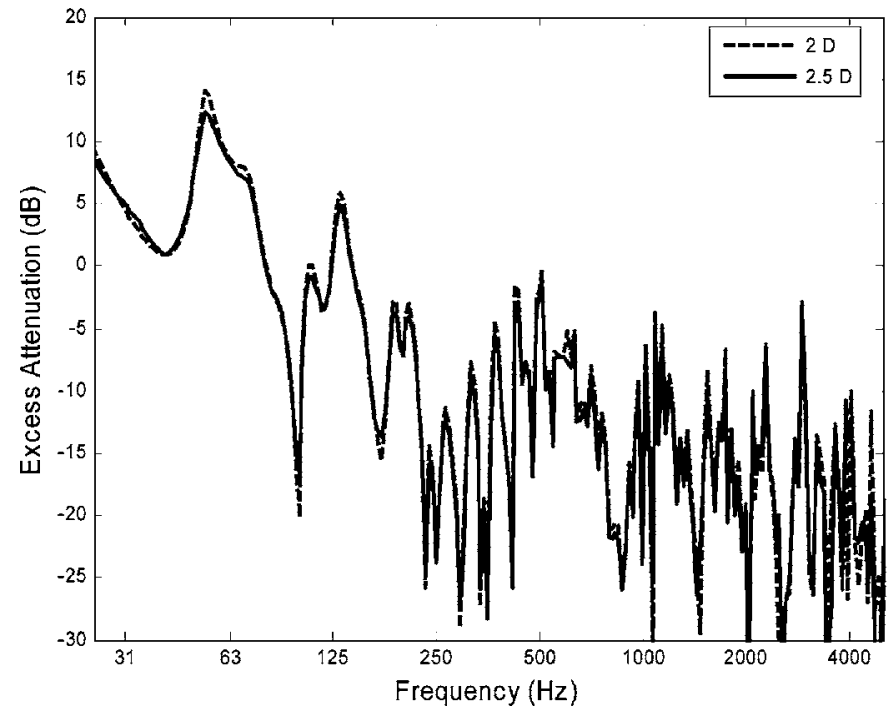

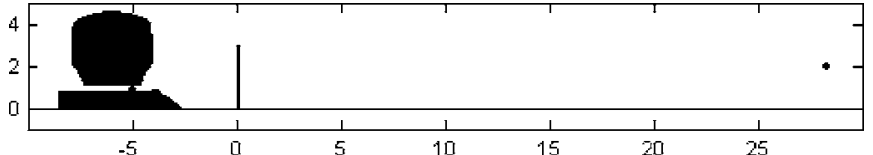

Fig. 13-Geometry of the configuration for an angle of $45^{\circ} . S(-4.95,0.85)$ and $R(28.14,2.0)$.

complete 3D models exist, they usually require significant running time. It is then of interest to use $2 \mathrm{D}$ models to reduce calculation times.

\subsection{Configuration and Results}

The 3D configuration is actually studied by performing 2D1/2 calculations (see Sec. 2.2). We study the sound propagation for a typical railway traffic geometry with a noise barrier to focus on body-barrier effects. The configuration is described in Fig. 10. The source is located at $0.1 \mathrm{~m}$ above the ballast which is representative of the wheel-rail contact. The receiver is located $20 \mathrm{~m}$ from the barrier and $2 \mathrm{~m}$ high above the ground. Barrier, ballast and train are assumed to be acoustically rigid to simplify the $3 \mathrm{D}$ calculation.

The two-dimensional approximation for oblique propagation over a barrier is illustrated in Fig. 11: a rotation of the barrier is carried out until it becomes perpendicular to the propagation path. Calculations are performed for $\theta=\arctan \left(y_{r} / x_{r}\right)$ equal to $0^{\circ}$ and $45^{\circ}$. At $0^{\circ}$, the same configuration is used in $2 \mathrm{D}$ and $2 \mathrm{D} 1 / 2$ case. Results are presented in Fig. 12. At $45^{\circ}$, the distance considered for 2D calculations are obtained by dividing the distance at $0^{\circ}$ by $\cos (\theta)$ (Fig. 13). Results are presented in Fig. 14.

(b)

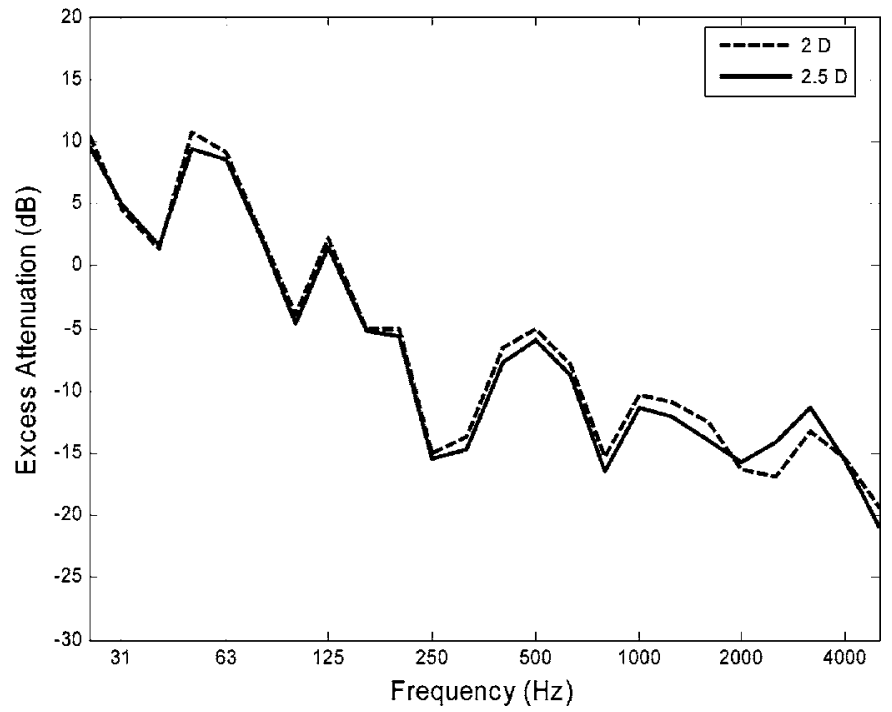

Fig. 12 - Excess attenuation versus frequency for $2 \mathrm{D}$ and $2 \mathrm{D} 1 / 2 \mathrm{BEM}$ calculations for $0^{\circ}$. Results in a) narrow bands and b) third octave band. 
(a)

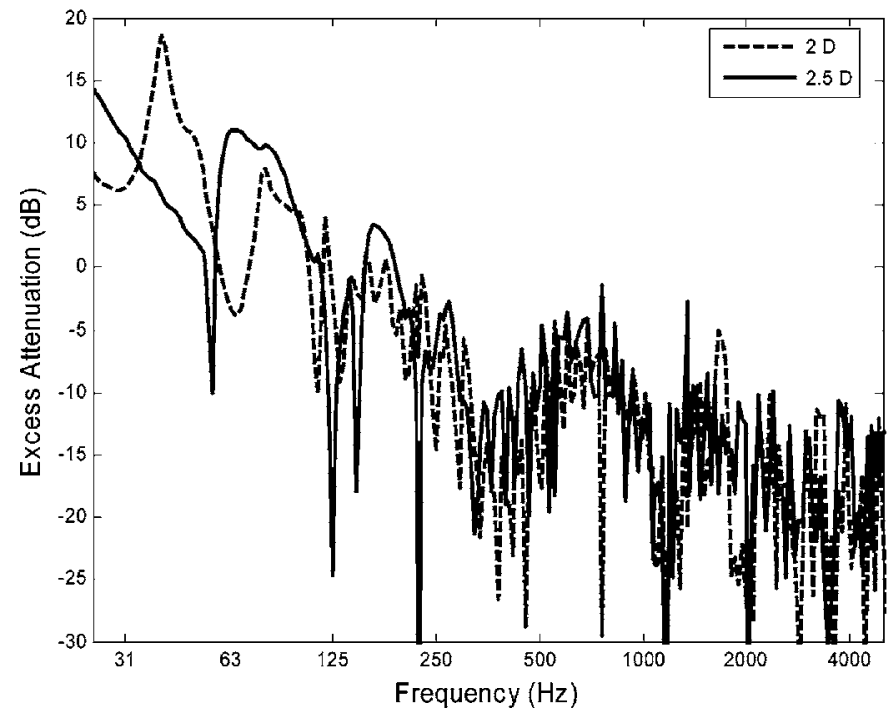

(b)

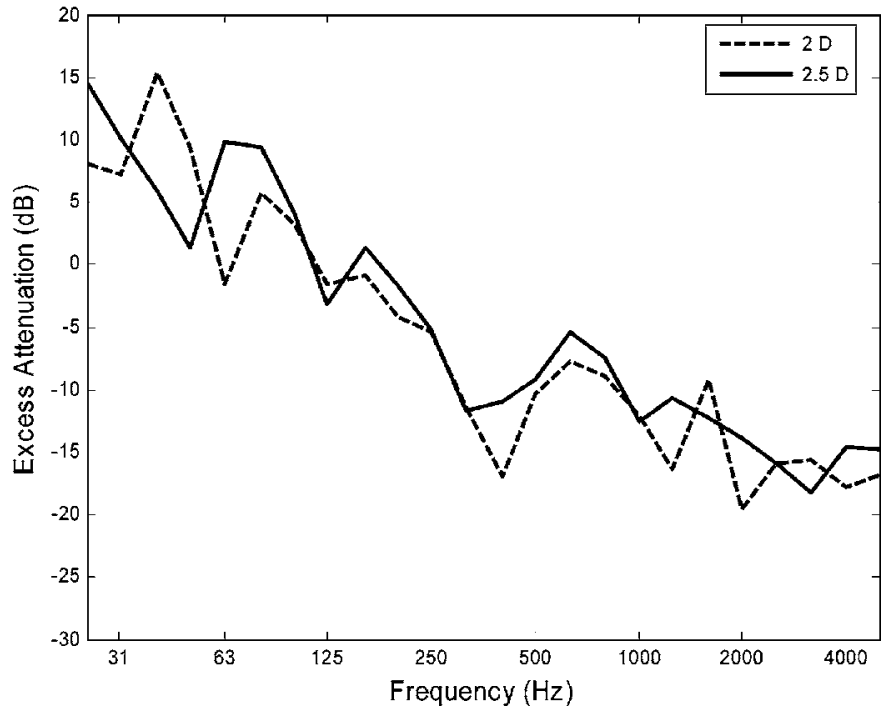

Fig. 14-Excess attenuation versus frequency for $2 \mathrm{D}$ and $2 \mathrm{D} 1 / 2$ BEM calculations for $45^{\circ}$. Results a) in narrow bands and $b$ ) third octave bands.

\subsection{Discussion and Conclusion}

There is close agreement between $2 \mathrm{D}$ and $2 \mathrm{D} 1 / 2$ results in the case of a propagation perpendicular to the railway track direction $\left(0^{\circ}\right)$. For the emission spectrum of a TGV rolling at $250 \mathrm{~km} / \mathrm{h}$ (Table 4), the difference in the A-weighted global excess attenuations calculated with a $2 \mathrm{D} 1 / 2$ and $2 \mathrm{D}$ approach is $0.4 \mathrm{~dB}$.

Unlike at $0^{\circ}$, the agreement between $2 \mathrm{D}$ and $2 \mathrm{D} 1 / 2$ calculations achieved at $45^{\circ}$ is not as good. However the global shape of the $2 \mathrm{D} 1 / 2$ excess attenuation spectrum is quite well predicted by the $2 \mathrm{D}$ approximation, especially in the frequency range $500-4000 \mathrm{~Hz}$ where the railway noise is dominant. Thus, for the emission spectrum of a TGV rolling at $250 \mathrm{~km} / \mathrm{h}$ (Table 4), the difference in the A-weighted global excess attenuation between $2 \mathrm{D} 1 / 2$ and $2 \mathrm{D}$ results does not exceed $0.9 \mathrm{~dB}$.

To conclude, the discrepancy between exact $2 \mathrm{D} 1 / 2$ and approximated 2D results is less than $1 \mathrm{~dB}$ in terms of the difference in the A-weighted global excess attenuation. This error is acceptable in most of studied cases. Furthermore, for the investigated configuration, the 2D calculations were about twice as fast as the 2D1/2 calculations. Therefore the approximation of $3 \mathrm{D}$ propagation planes by $2 \mathrm{D}$ equivalent ones may be used with confidence in the reference model.

\section{LINEARIZATION OF SOUND SPEED PROFILES}

\subsection{Introduction}

Sound speed profile $c$ above real road traffic noise configuration may usually be approximated by a logarithmic function:

$$
c(z)=c_{0}+b \ln \left(1+\frac{z}{z_{0}}\right)
$$

where $c_{0}$ is the reference sound speed, $b$ is the refraction parameter, $z_{0}$ the roughness length and $z$ the height. However a linear sound speed profile is used in many reference models:

$$
c(z)=c_{0}(1+a z)
$$

where $a$ is refraction index. The objective here is to quantify the error due to this linear approach. Calculations are achieved in inhomogeneous atmosphere using ATMOS.

\subsection{Basis of the Linearization of Sound Speed Profiles}

The linear profile is evaluated as an average sound speed profile using a Fresnel volume approach. ${ }^{18}$ The sound speed profile can be estimated as a function of the average propagation height $h_{m}$ and the maximum width of the Fresnel ellipse $h_{f}$ (Fig. 15):

$$
h_{m}=\frac{z_{s}^{2}+z_{r}^{2}}{2\left(z_{s}+z_{r}\right)}
$$

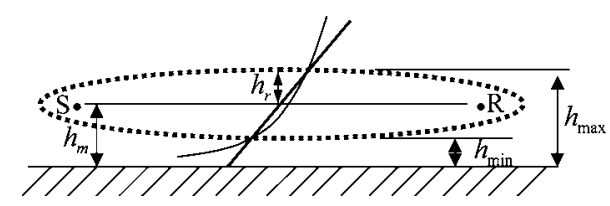

Fig. 15-Principle of the equivalent sound speed profile with the Fresnel volume approach. 
Table 4-Emission spectrum of a TGV rolling at $250 \mathrm{~km} / \mathrm{h}$.

\begin{tabular}{cccccccccc}
\hline \hline Frequency & $100 \mathrm{~Hz}$ & $125 \mathrm{~Hz}$ & $160 \mathrm{~Hz}$ & $200 \mathrm{~Hz}$ & $250 \mathrm{~Hz}$ & $315 \mathrm{~Hz}$ & $400 \mathrm{~Hz}$ & $500 \mathrm{~Hz}$ & $630 \mathrm{~Hz}$ \\
$\mathrm{~L}(\mathrm{~dB})$ & $-45,3$ & $-48,3$ & 51 & 50,7 & 53 & 55 & 57,2 & 58,8 & 60,1 \\
Frequency & $800 \mathrm{~Hz}$ & $1000 \mathrm{~Hz}$ & $1250 \mathrm{~Hz}$ & $1600 \mathrm{~Hz}$ & $2000 \mathrm{~Hz}$ & $2500 \mathrm{~Hz}$ & $3150 \mathrm{~Hz}$ & $4000 \mathrm{~Hz}$ & $5000 \mathrm{~Hz}$ \\
$\mathrm{~L}(\mathrm{~dB})$ & 62,2 & 63 & 63,6 & 66 & 66,2 & 66,3 & 62,6 & 62,5 & 61,9 \\
\hline \hline
\end{tabular}

$$
h_{f}=\sqrt{\frac{\lambda}{4}}\left(r+\frac{\lambda}{4}\right)
$$

where $\lambda$ is the wave length, $r$ the distance between source and receiver, $z_{s}$ the source height and $z_{r}$ the receiver height.

Thus the average sound speed profile is determined between the heights $h_{\min }$ and $h_{\max }$ of the ellipse by calculating $a$ as:

$$
a=\frac{c\left(h_{\max }\right)-c\left(h_{\min }\right)}{c_{0} \cdot\left(h_{\max }-h_{\min }\right)}
$$

with $h_{\max }=h_{m}+h_{f}, h_{\min }=h_{m}-h_{f}, c_{0}$ being the reference sound speed, and $c\left(h_{\min }\right)$ and $c\left(h_{\max }\right)$ are two effective sound speeds at heights $h_{\min }$ and $h_{\max }$ :

$$
\begin{aligned}
& c\left(h_{\text {min }}\right)=c_{0}+b \ln \left(1+\frac{h_{\text {min }}}{z_{0}}\right) \\
& c\left(h_{\text {max }}\right)=c_{0}+b \ln \left(1+\frac{h_{\text {max }}}{z_{0}}\right)
\end{aligned}
$$

When the source and/or the receiver are close to the ground, $h_{\text {min }}$ becomes negative $\left(h_{m}<h_{f}\right)$. For such cases $h_{m}$ keeps a minimal positive value $h_{m}=2 \cdot h_{0}$ where $h_{0}$ is the average roughness length of the ground $\left(h_{0}\right.$ $=z_{0} / 0.15$ ).

We consider here a sound speed profile creating by a wind. Wind is fluctuating and irregular phenomena so that its speed needs to be modeled statistically. Thus, the instantaneous wind speed $u(t)$ is split into an average wind speed $\bar{u}$ and a fluctuating part $u^{\prime}(t)$. So that:

$$
u(t)=\bar{u}+u^{\prime}(t)
$$

where $t$ represents the time.

We are interested in considering a period of about one year. For such a long-term study the wind variation to be investigated is no more the fluctuating part $u^{\prime}(t)$ but the average speed $\bar{u}$ itself. We use the Weibull law ${ }^{19}$ to define the wind speed distribution, $p_{w}(\bar{u})$. It is given by:

$$
p_{w}(\bar{u})=\frac{K}{\bar{u}}\left(\frac{\bar{u}}{A}\right)^{K} \exp \left(-\left(\frac{\bar{u}}{A}\right)^{K}\right)
$$

where $K$ is a shape parameter, $\bar{u}$ the average wind speed and $A$ the scale parameter. The law for a wind speed of
$2 \mathrm{~m} \cdot \mathrm{s}^{-1}$ at $10 \mathrm{~m}$ above the ground is presented in Fig. $16\left(K=1.8\right.$ and $\left.A=2 \mathrm{~m} \cdot \mathrm{s}^{-1}\right)$.

A set of excess attenuations $E A_{i}$ is computed for several wind speeds $u_{i}$. The average excess attenuation frequency is then obtained by associating each excess attenuation $E A_{i}$ with its occurrence probability $p_{w i}$ :

$$
E A_{a v}(f)=\frac{\sum_{i} E A_{i}(f) p_{w i}}{\sum_{i} p_{w i}}
$$

where $f$ is the frequency.

\subsection{Results}

The source is located $0.5 \mathrm{~m}$ high above ground. A $2 \mathrm{~m}$ high receiver is located $250 \mathrm{~m}$ from the source. Excess attenuation computed with a logarithmic sound speed profile is compared with excess attenuation computed with a variable linear sound speed profile and excess attenuation computed with long term logarithmic sound speed profile is compared with excess attenuation computed a variable linear sound speed profile averaged over the same period (Fig. 17).

\subsection{Discussion and Conclusion}

Comparison between excess attenuation computed with a logarithmic sound speed profile and with a

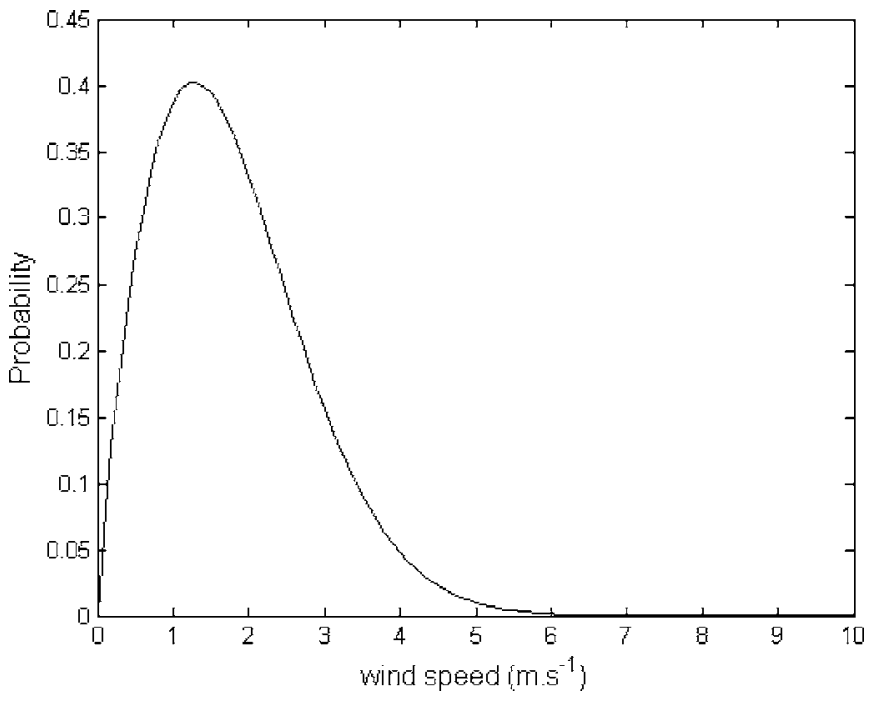

Fig. 16-Wind speed repartition with the Weibull law. 
(a)

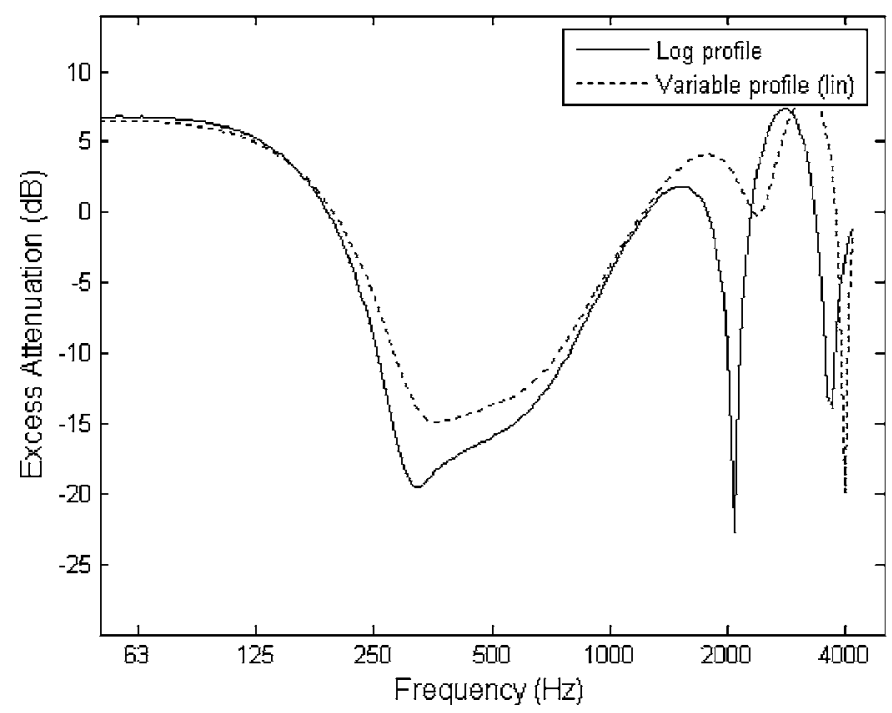

(b)

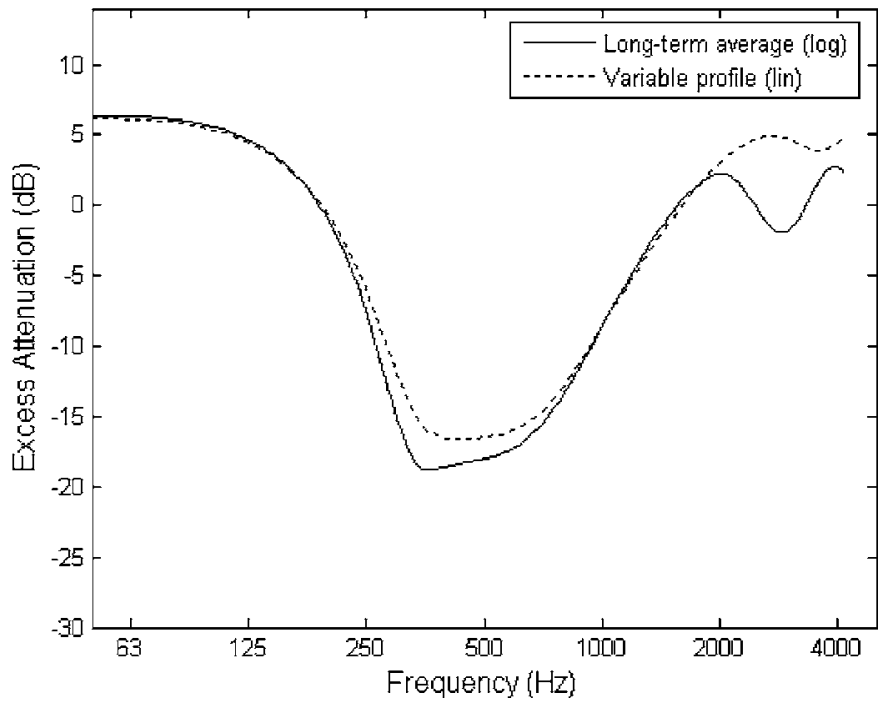

Fig. 17-Excess Attenuation a) for a logarithmic sound speed profile and b) for an average long-term logarithmic sound speed profile with a variable linear sound speed profile.

variable linear sound speed profile points out an important deviation, especially at interferences location (Fig. 17(a)). Nevertheless, results obtained for long-term calculations with logarithmic sound speed profiles are much the same as results achieved with variables linear sound speed profiles (Fig. 17(b)). In our investigated case, the discrepancy between the two methods is about $0.1 \mathrm{~dB}$ in terms of difference of the A-weighted global attenuation for a normalized traffic noise spectrum ${ }^{16}$ (Table 4).

The linearization of sound speed profiles can be an efficient alternative to introduce average long-term logarithmic sound speed profile in a reference model. This approach can be used to introduce linear equivalent meteorological effects in BEM calculations using the analogy between sound propagation above a flat surface along curved ray paths and sound propagation above a curved surface along straight ray paths. ${ }^{20}$

\section{CONCLUSION}

This paper presents five approaches which could be used to reduce the complexity of an outdoor sound propagation study. They deal with different effects related to ground, multiple-reflections, wind over barriers, oblique propagation over barriers and linearization of sound speed profile. Each principle has been investigated and discussed separately for a typical road or railway configuration. Except for the approximation of wind speed near barriers, the use of these assumptions gives accurate results with a difference in an A-weighted global excess attenuation lower than $1 \mathrm{~dB}$ in contrast to the reference case. Therefore, they can be used with confidence. Some of them can be useful in reducing high calculation times (ground effect, multiple reflections order for barrier, two-dimensional approximation for oblique propagation over barrier) or to extend the application field of some reference models (ground effect, two-dimensional approximation for oblique propagation over a barrier, or linearization of a logarithmic sound speed profile).

The topic of approximating the wind speed near barriers points out the importance of taking the barrier effect on wind into consideration. When this assumption is used for calculations, it produces an error above $3 \mathrm{~dB}$ in an A-weighted global excess attenuation. The calculations have been performed here without any turbulence. Further analysis should be conducted with turbulence.

\section{REFERENCES}

1. F. Aballéa and J. Defrance, "Sound Propagation Over Irregular Terrain With Complex Meteorological Effects Using the Parabolic Equation Model," Proc. Internoise. Prague, Czech Republic (2004).

2. F.-E. Abelléa, "Outdoor sound propagation: Application of the fast parabolic equation to meteorological effects and complex topographies (in French)," Thesis, Université du Maine, Le Mans, France (2004).

3. P. Jean, "A variational approach for the study of outdoor sound propagation and application to railway noise," J. Sound Vib. 212(2), 275-294 (1998).

4. HARMONOISE, European project IST 2000-18419 - Reference Model - Task 2.5 - Modeling solutions and algorithms (2003).

5. K. Attenborough, S. Taherzadeh, H. E. Bass, R. Rasped, G. R. Becker, A. Gudesen, A. Chrestman, G. A. Daigle, A. L'Esperance, Y. Gabillet, Y. Gabillet, Y. L. Li, M. J. White, P. Naz, J. M. Noble, and H. A. J. M. Van Hoff, "Benchmark cases for outdoor sound propagation models," J. Acoust. Soc. Am. 97(1), 173-191 (1995). 
6. M. Galindo, M. R. Stinson, and G. A. Daigle, "Comparison of some methods used for prediction of atmospheric sound propagation," Canadian Acoustics, 25(4), 3-11 (1997).

7. K. E. Gilbert and M. J. White, "Application of the parabolic equation to sound propagation in refracting atmosphere," $J$. Acoust. Soc. Am. 85(2), 630-637 (1989).

8. K. E. Gilbert and X. Di, "A fast Green's function method for one-way sound propagation in the atmosphere," J. Acoust. Soc. Am. 94(4), 2343-2352 (1993).

9. X. Di and K. E. Gilbert, "Application of a fast Green's function method to long range sound propagation in the atmosphere," Proc. 5th International Symposium on Long Range Sound Propagation (1992).

10. E. M. Salomons, "Improve Green's function parabolic equation method for atmospheric sound propagation," J. Acoust. Soc. Am. 104(1), 100-111 (1998).

11. N. Barriere and Y. Gabillet, "Sound propagation over a barrier with realistic wind gradients. Comparaison on wind tunnel experiment with GFPE computation," Acust. Acta Acust. 85, 325334 (1999).

12. R. D. Ciskowski and C. A. Brebbia, Boundary Element Methods in Acoustics, Elsevier Applied Science, London (1991).

13. D. Duhamel, "Efficient calculation of the three-dimensional sound pressure field around a noise barrier," J. Sound Vib. 197(5), 547-571 (1996).

14. P. Jean, J. Defrance, and Y. Gabillet, "The importance of source type on the assessment of noise barriers," J. Sound Vib. 226(2), 201-216 (1999).

15. M. E. Delany and E. N. Bazley, "Acoustical properties of fibrous absorbent materials," Appl. Acoust. 3, 105-116 (1970).

16. Acoustics - Road traffic noise reducing devices - Test method for determining the acoustic performance - Part 3: Normalized traffic noise spectrum, European Standard EN 1793-3: 1997, European Committee for Standardization (1997).

17. F. Aballéa and J. Defrance, "Simple and multi-reflections using the PE method with a complementary Kirchhoff approximation," Proc. 7ème Congrès Français d'Acoustique. Strasbourg, France (2004).

18. A. L'Esperance, "Modeling sound wave propagation in a natural complex environment (in French)," Thesis, Sherbrooke University, Sherbrooke (1992).

19. C. Sacre, Physics of Buildings Handbook (in French), CSTB, Paris (1995).

20. E. Premat and J. Defrance, "Theoretical and experimental study of sound propagation for traffic noise," Proc. 9th ICSV. Orlando (2002). 